\title{
Fruit Quality Analysis using Image Processing
}

\author{
K B Mirra, P Pooja, S Ranchani, R Rajakumari
}

\begin{abstract}
Fruit Analysis using image processing is a technique used for finding the detection of fruits by a specific algorithm. This project is also used in finding the defected fruit in a group of fruits by the image processing technique. The objective of this work is to prevent health hazards by eating that defected fruit. This processing technique undergoes several stages for the classification and prediction of the defective fruit. This paper presents a detailed overview of various methods i.e., preprocessing, segmentation, feature extraction, a designation that addressed fruits and vegetable quality based on color, texture, size, shape, and defects. In this paper, a critical comparison of a different algorithm proposed by researchers for quality inspection of fruits has been carried out.
\end{abstract}

Keywords: Fruit grading, Image processing, classification, Quality analysis.

\section{INTRODUCTION}

In representing the conception for the human brain, images are the most basic method in the physical classification of foodstuff. Factors affecting fruits can be quantified visually, which is laborious, expensive, and is easily affected by physical factors, including the inconsistent evaluation and subjective results. The market prices are determined by such inspections and, also, the "best-if-used-before date." The trained human investigators have done the quality inspection by feeling and seeing. This method is significantly inconsistent, fickle, and decisions are seldom the same among investigators. The computer vision system and image processing are readily growing research area, which is a significant analyzing technique for fruit analysis.

\section{RELATED WORKS}

Aleixos et al. designed and implemented a multispectral camera to capture visible and near infrared images [1]. The main focus was on size, color and presence of defects on citrus fruits. The given experiment was able to inspect the

Revised Manuscript Received on May 15, 2020.

* Correspondence Author

K.B Mirra*, Computer Science and Engineering, National engineering college, Kovilpatti, India. Email: 1612063@nec.edu.in

P. Pooja, Computer Science and Engineering, National engineering college, Kovilpatti, India. Email: 1612075@nec.edu.in

S.Ranchani, Computer Science and Engineering, National engineering college, Kovilpatti, India. Email: 1612082@nec.edu.in

R Rajakumari, Computer Science and Engineering, National engineering college, Kovilpatti, India.

(c) The Authors. Published by Blue Eyes Intelligence Engineering and Sciences Publication (BEIESP). This is an open access article under the CC BY-NC-ND license (http://creativecommons.org/licenses/by-nc-nd/4.0/) lemons, mandarins and oranges.The study presents a parallel system for estimating size as well as inspecting its surface for diseases. Proposed system was capable of classifying mandarins and lemon correctly with the precession of $93 \%$. Kleynen et al. described the best filters for detecting broad range of wavelength for detecting defects on fruit based upon quadratic discriminant analysis. Multi spectral vision system was used for this work. NIR spectrometer was used for reflectance spectra of damaged and sound surface [2]. Defects considered were scald, visible flesh damage, bruises, frost damage etc. For differentiating bruise surface from healthier one was performed by the use of combination of four filters. Thus the proposed method was able to select the wavelength band to sort the jonagold apples.

Leemans and Destain has presented a grading method for jonagold apples only. Technique used in this approach was k-means there were various steps that performed for grading the jonagold apples. First image acquisition was performed and then segmentation of image was done to locate the fruit in the image. Finally find the possibility of defects for the grading purpose. The correct rate of classification for jonagold apple was $73 \%$.

Brewer et al. had developed the analyzer for tomato fruit and other descriptors were combined. Where combined Vocabulary defines bounds for species of fruits shape and mathematical descriptor can measure the fruit feature with a single formula for each feature. Such features were shape of fruit, index of fruit species of plant. So that to analyze the shape and size of tomato and other species of plant. While other species of plant may include banana, pepper, grape, strawberry and chili pepper [4]. Different colors were used for determining the boundaries of fruit accurately. The researcher could implement recognize the shape variation of tomato and depict the 2-D shape of fruit. It provides the analysis of morphology of fruit and gives descriptors. For achieving the objective of measurement of shape of fruits, the controlled vocabulary and mathematical shape, fruit shape triangle, eccentricity of fruit shape, circular, rectangular and ellipsoid. Fruit size includes height, width, area, mass and perimeter of fruit. Roushdy described a subjective investigation of edge recognition strategies were described. Experimental results demonstrate that Boie-Cox, Shen- Castan and Canny operators were superior to Laplacian of Gaussian (LOG), while LOG was superior to Prewitt furthermore, Sobel if there was need to deal with noisy image. This was because Gaussian edge recognizers are symmetric on the edge and noise can minimize by image smoothing [5]. Subjective and objective techniques were utilized to compare the diverse edge operators. The morphological filter was more vital as an introductory process in the edge location for noisy image and utilized opening-closing operation as pre-processing to filter noise. Additionally,

Published By:

Blue Eyes Intelligence Engineering

\& Sciences Publication

(C) Copyright: All rights reserved. 
smooth the image by first closing and then dilation to improve the image before the edge operators ${ }^{e e}$ influence. Blasco et al. proposed an algorithm for fruit sorting including mandarins and oranges. It also present system in which recognition and classification of skin defects of citrus fruit was performed. Techniques used for overall distinguish of defect were near infrared reflectance (NIR) and ultraviolet induced fluorescence (UVFL) [6].

With the use of morphological operations, a fruit sorting algorithm was proposed.

A fruit sorting algorithm was developed by the contribution of NIR and UVFL. The success rate of classification was $86 \%$.

Liming and Yancho had graded the strawberry based on image processing techniques. Features used for grading are shape, size and color.

Uemura et al. had presented an edge detection approach. Edge detection was the basic and important step for efficient image segmentation.

\section{PROPOSED WORK}

Quality has become a vital aspect for the consumer to differentiate the products of the same category. Nowadays, the consumer's viewpoint for quality checking is essential. Especially, in the case of the food industry, dealers take care of fruits as these products are directly fed from farm to customer through many stages such as packing, transportation, etc. The possibility of food products getting spoiled is likely to happen because of fruit harvesting, preparation, and supplying of fruits so that different dealers can provide fruits to consumers. This whole process takes a long time. Hence, there is a need for a fast and efficient algorithm for quality identification. For the essence of fruit commerce, manufacturers have to produce cost-effective refined products. Traditionally, quality is investigated manually, which is a boring and time-consuming activity.

A larger number of workers are required in this process, but there is a labor shortage problem Moreover, long shifts are required with limited workers. Working conditions are hard. The most important thing is the limitation of time for product freshness. These problems lead to developing an efficient algorithm for recognition and quality identification of fruits encompassing image processing techniques. It may affect the quality of the product since it will enhance the speed and decrease the manual errors. An algorithm will take an image of fruit then extract the fruit from that image using image processing techniques such as segmentation is required. Further, a set of the procedure has to follow for quality identification of a fruit, which will notify the quality as good or harmful to the user.

\section{Training Data}

In this process, the collection of images takes place, and the photos consist of lemon, orange, and tomato. Here we collect the image of the usual lemon, orange, and tomato. Then we also collect the rotten images of lemon, orange, and tomato. With the help of these images, it undergoes a preprocessing technique. Firstly these images should be undergone several processes and training the system should be taken place. Then only we can able to find the defective fruit easily.

\section{Color identification}

Here the identification of the fruit color takes place. With the help of this color, we can able to classify the fruits easily. The defective fruit can be identified by their color, size, texture, and shape. Classification is done using the SVM algorithm. The KNN algorithm also used to find defective fruit.

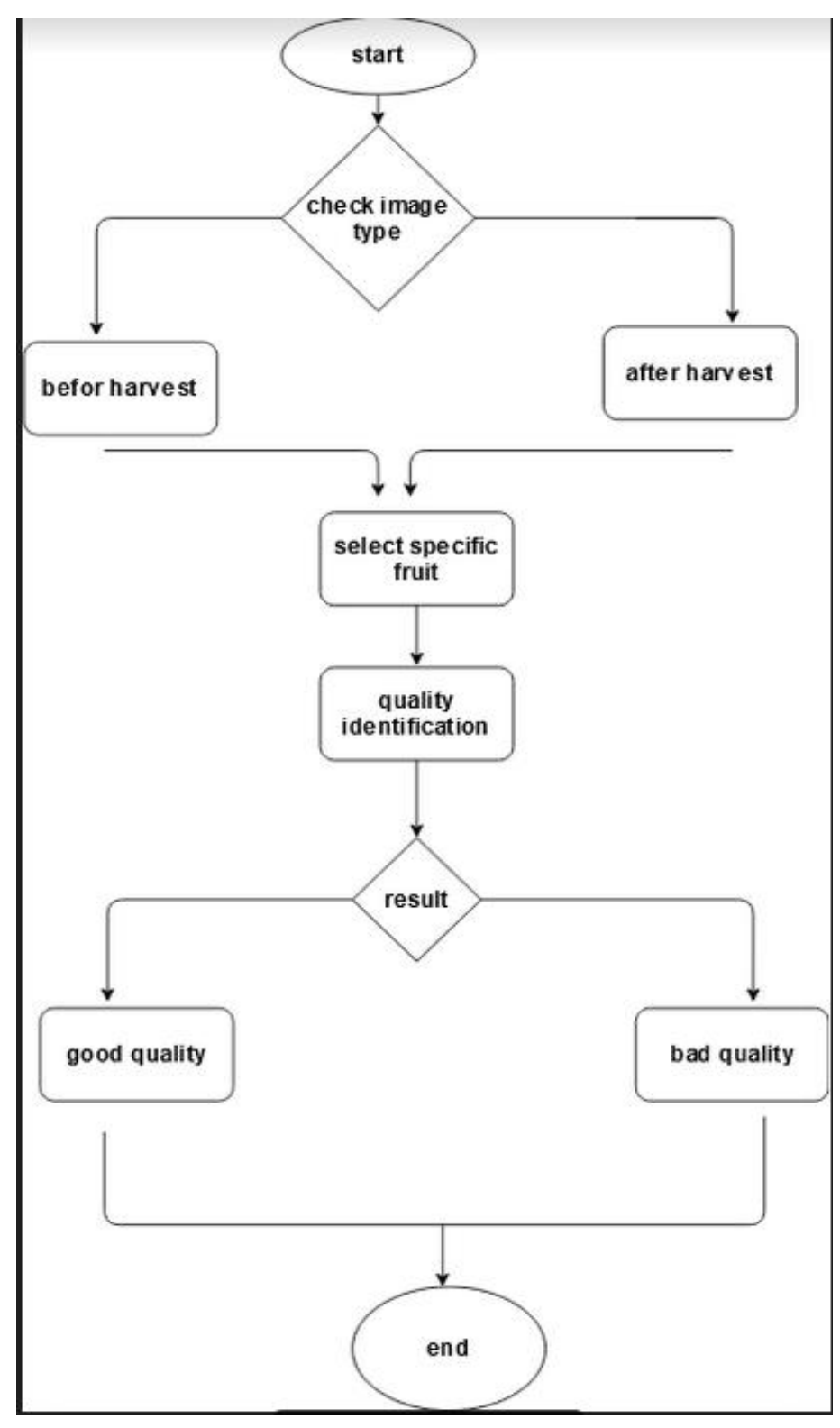

Figure -1: flow chart diagram

\section{A. Preprocessing}

Images are collected, then it moves on to the preprocessing stage in here training of data take place i.e., reduce the noise and size of the image.

\section{B. Feature Extraction}

The next stage is the feature extraction. Here, the feature will be extracted in a precise way. Features such as Contrast, Correlation, autocorrelation, Cluster Prominence, Dissimilarity, Energy, Entropy, Homogeneity.so thereby, the defection of fruits can be easily collected.

The next stage is the segmentation here. It is done by the SVM algorithm. Support vector are machines supervised learning models with associated learning algorithms that analyze data used

Published By:

Blue Eyes Intelligence Engineering \& Sciences Publication

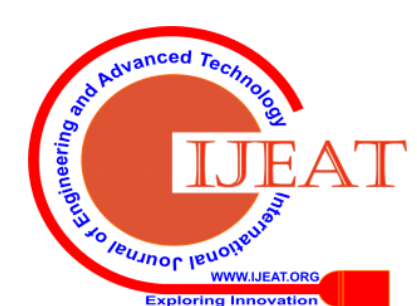


for classification and regression analysis. Given a set of training examples, each marked as belonging to one or the other of two categories, an SVM training algorithm builds a model that assigns new examples to one category or the other, making it a non-probabilistic binary linear classifier.

\section{Classification}

The next stage is the classification. here classification is done by the KNN Algorithm K-Nearest Neighbors (k-NN) is a supervised machine learning algorithm i.e. it learns from a labelled training set by taking in the training data $\mathrm{X}$ along with its labels $y$ and learns to map the input $X$ to its desired output $Y$. The model only consists of the training data, that is, the model simply learns the entire training set and for prediction gives the output as the class with the majority in the ' $\mathrm{k}$ ' nearest neighbors calculated according to some distance metric. After the model has stored the training set for prediction, it takes a test image to be predicted, calculates the distance to every image in the training set and obtains the ' $\mathrm{k}$ ' training images closest to the test image. It then outputs the class according to some voting procedure from the labels of these ' $k$ ' neighbors, generally a majority vote.The distance metric used to calculate distances may differ, such as either a L1 distance function which is the summation of the differences between the pixels of the images.

$$
d 1(I 1, I 2)=\sum p|I p 1-I p 2|
$$

So thereby defected fruits are classified and can be found easily through this stage.

\section{Identification}

In MatLab identification, fruit can be done by this stage, and here it will display the defected fruit and fruit which are not defective also can be identified through this stage.

\section{RESULT AND DISCUSSION}

There are five Sections for the fruit quality detection in proposed methodology. These steps and the results are shown below.

Section 1: The images of Orange which are before trained.

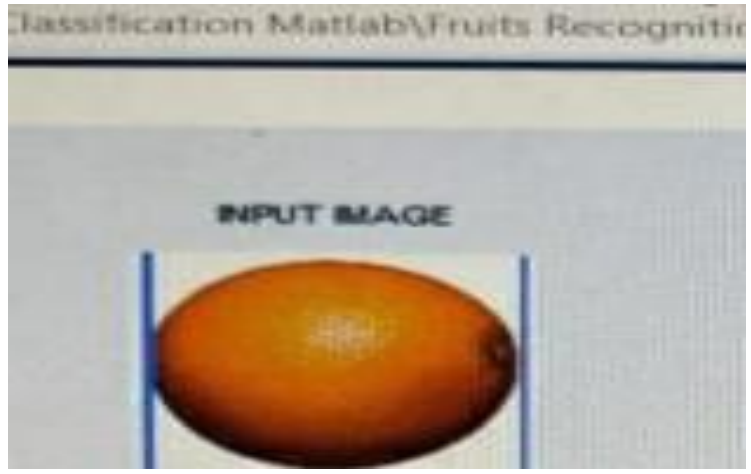

Figure -2: Input image

Section 2: The input image of orange is converted into gray image.

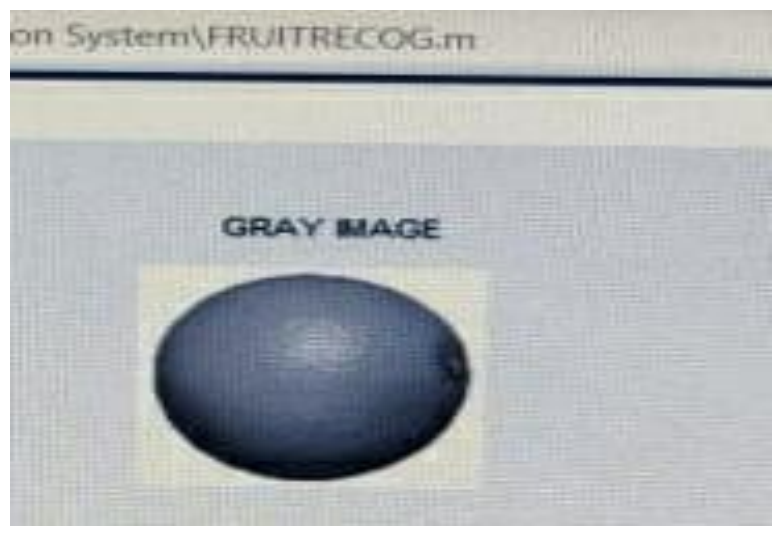

Figure -3: Gray image

Section 3: Here we have the training data images.

For training, the system has trained set of 50 images. For testing, the system has tested 50 images. Training and testing process are shown in Figure-4 and Fogure-5.

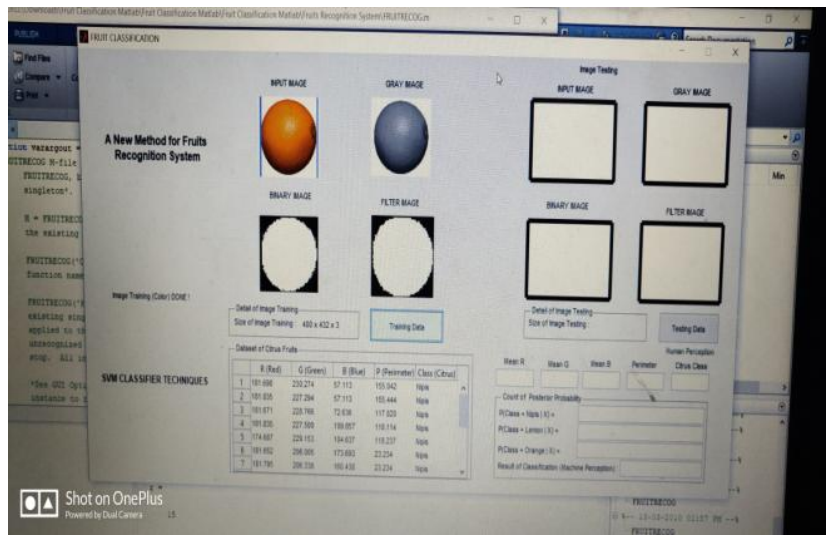

Figure -4: Training Image

Section 4: Here we test for the good quality fruit and it will display the fruit image.

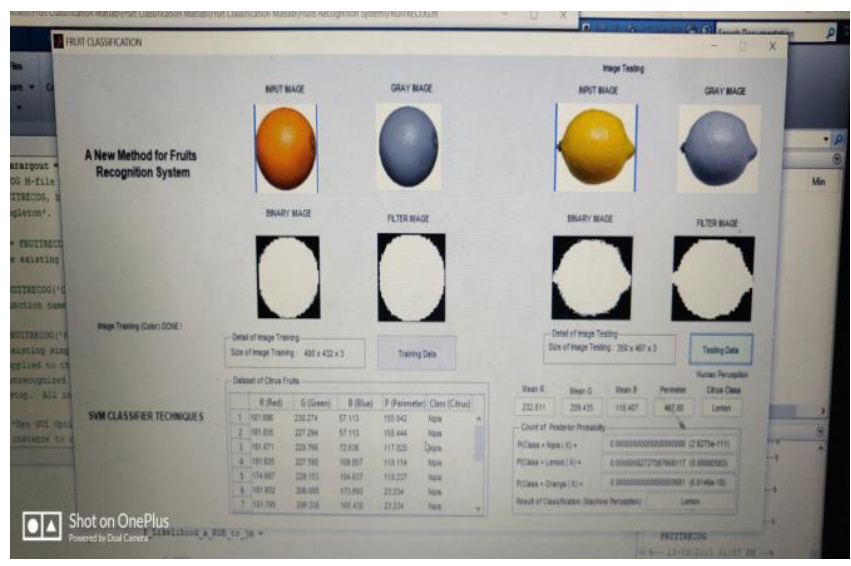

Figure -5: Fruit image

Section 5: shows the detection of fruit

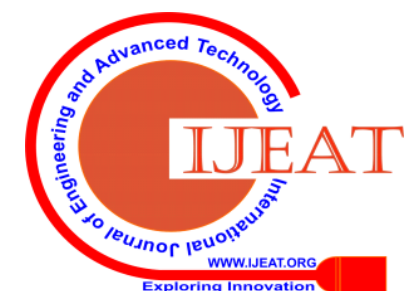




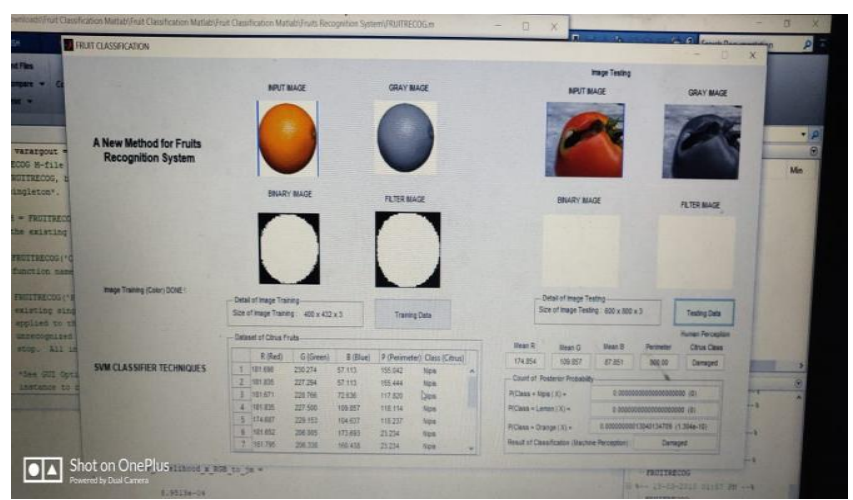

Figure -6: Fruit Defection

\section{CONCLUSION}

Surface defects are the essential trait of fruits, which not only affect their market value but purchasers ${ }^{\text {ee }}$ inclination to their inward quality also to some extent. Therefore, it is essential and important to get fruit without defects; otherwise, it becomes a cumbersome task at the post-harvesting stage. Inadequate manual investigation will require a lot of labor and time. In the present work, quality identification of fruit is made by using the proposed algorithm for the segmentation of fruit. Total of 50 sample images of fruit. Where 50 images of each type (good and bad quality) are taken for testing. Different segmentation methods are used for images of lemon. For the image of fruits, the segmentation of orange is done based upon its color and shape. After the segmentation, single fruit is selected by the user. A further feature is extracted from selected fruit by utilizing the GLCM approach. Different classifiers in the present study have been used to the classification of fruit into good or bad quality. After this, the results of different classifiers are compared. Results demonstrate that $89.16 \%$ accuracy of classification has achieved by using an SVM classifier. However, the KNN classifier shows a preferable result over SVM with an accuracy of $93.33 \%$.

\section{REFERENCES}

1. Aleixos N., Blasco J., Navarron F. and Moltó E., "Multispectral Inspection of Citrus in Real-Time using Machine Vision and Digital Signal Processors",Computers and electronics in agriculture, vol. 33, no. 2, pp. 121-137, 2002.

2. Kleynen O., Leemans V. and Destain M. F., "Selection of the Most Efficient Wavelength Bands for „Jonagold "e Apple Sorting", Postharvest Biology and Technology, vol. 30, no. 3, pp. 221-232, 2003.

3. Leemans V. and Destain M. F., "A Real-Time Grading Method of Apples Based on Features Extracted from Defects", Journal of Food Engineering, vol. 1, no. 1, pp. 83-89, 2004.

4. Brewer M. T., Lang L., Fujimura K., Dujmovic N., Gray S. and van der Knaap E., "Development of A Controlled Vocabulary And Software Application to Analyze Fruit Shape Variation in Tomato And Other Plant Species", Plant Physiology, vol. 141, no. 1, pp. 15-25, 2006.

5. Roushdy M., "Comparative Study of Edge Detection Algorithms Applying on The Grayscale Noisy Image Using Morphological Filter", GVIP journal, vol. 6,no. 4, pp. 17-23, 2006.

6. Gómez-Sanchis J., Moltó E., Camps-Valls G., Gómez-Chova L., Aleixos N. and Blasco J., "Automatic Correction of the Effects of the Light Source on Spherical Objects. an Application to the Analysis of Hyperspectral Images of Citrus Fruits", Journal of Food Engineering, vol. 85, no. 2, pp. 191-200, 2008.

7. Blasco J., Aleixos N., Gómez-Sanchis J. and Moltó E., "Recognition And Classification of External Skin Damage in Citrus Fruits Using Multispectral Data And Morphological Features", Biosystems engineering, vol. 103, no. 2, pp.137-145, 2009.

8. Bashish A. D., Braik M. and Bani-Ahma S., "A Framework for
Detection And Classification of Plant Leaf and Stem Diseases", In Proceedings of InternationalConference on In Signal and Image Processing (ICSIP), pp. 113-118, 2010.

9. Liming X. and Yanchao Z., "Automated Strawberry Grading System Based on Image Processing", Computers and Electronics in Agriculture, vol. 71, pp. S32-S3,2010.

\section{AUTHORS PROFILE}

R. Rajakumari is working as a Senior Assistant Professor in Computer Science \& Engineering at National Engineering College, Kovilpatti. She has more than eleven years of teaching and research experiences. She has attended more than five international conferences and authored seven international journals. Her area of specialization is pattern recognition and she is doing research in medical image processing. She is a Life member in ISTE society.

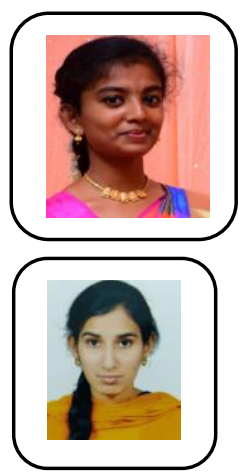

K.B. Mirra is currently pursuing her final year Bachelor of Engineering in Computer Science. She Has done a project Time table generator using php \& mysql. She is a member in IEEE society. Her area of interest is cloud computing.

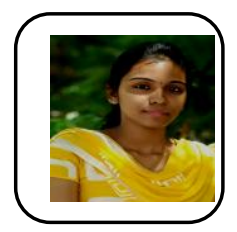

S. Ranchani is currently pursuing her final year Bachelor of Engineering in Computer Science. She has done a project Time table generator using php \& mysql. She is a member in SMT society. Her area of interest is Java.

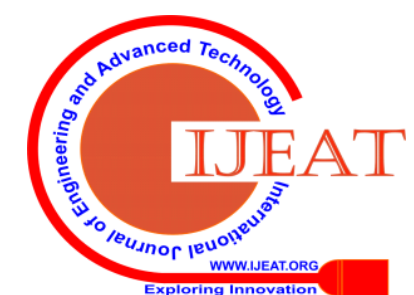

